\section{Responsabilidad social corporativa en instituciones financieras del Ecuador}

\author{
Corporate social responsibility in financial institutions in Ecuador
}

Bélgica Cecilia Arias Macías; Carmen Luisa Soto Montoya

\section{RESUMEN}

La incorporación de la Responsabilidad Social Corporativa (RSC) en Ecuador, es un tema de reciente interés. En esta problemática se embarca el sistema financiero ecuatoriano que busca orientar sus actividades considerando las expectativas de los diferentes grupos de interés afectados por sus acciones para contribuir al desarrollo sostenible. El objetivo de este documento es realizar un análisis comparativo de la responsabilidad social corporativa de las instituciones financieras (IF) del Ecuador. Por esta razón se toma de referencia tres de las cuatro IF consideradas como grandes, al ser estas las que aplican las normas de responsabilidad social de Global Reporting Initiative (GRI 4) e ISO2600o. Sus memorias permitieron la aplicación de un instrumento de medición. Para la categoría de economía se realizó la comparación de sus balances donde destaca la IF que generó mayor rentabilidad a sus inversionistas. En la categoría social, se mide la percepción de sus colaboradores y destaca aquella institución donde estos perciben un mejor ambiente laboral. Y, por último, en la categoría medio ambiente se analizó los datos de las memorias de las tres instituciones y por medio de un check list basado en las GRI 4 se determinó la institución que más cumple con los índices de RSC.

Palabras clave: Responsabilidad social; instituciones financieras; dimensiones económicas; social y ambiental

\section{ABSTRACT}

The incorporation of Corporate Social Responsibility (CSR) in Ecuador is a topic of recent interest. The Ecuadorian financial system embarks on this problem, which seeks to guide its activities considering the expectations of different interest groups affected by its actions to contribute to sustainable development. The objective of this document is to carry out a comparative analysis of the corporate social responsibility of financial institutions in Ecuador. For this reason, three of the four Fls considered as large are taken as a reference, as these are the ones that apply the Global Reporting Initiative and ISO26000 social responsibility standards. His memories of social responsibility allowed the application of a measuring instrument. For the economy category, a comparison of their balance sheets was made, highlighting the FI that generated the highest profitability for its investors. In the social category, the perception of their collaborators is measured and the institution where they perceive a better work environment stands out. And finally, in the environment category, the data from the reports of the three institutions were analyzed, and by means of a checklist based on the Global Reporting Initiative, the institution that most complies with the CSR indices was determined.

Keywords: Social responsibility; financial institutions; economic dimensions; social and environmental

\section{RELIGACIÓN \\ REVISTA DE CIENCIAS SOCIALES Y HUMANIDADES JOURNAL OF SOCIAL SCIENCES AND HUMANITIES
REVISTA DE CENCIAS SOCIAIS E HUMANAS}

INFORMACIÓN:

http://doi.org/10.46652/rgn.v6i30.870 ISSN 2477-9083

Vol. 6 No. 30, 2021. e210870 Quito, Ecuador

Enviado: octubre 20, 2021

Aceptado: diciembre 14, 2021

Publicado: diciembre 20, 2021

Publicación Continua

Sección Sur-Sur| Peer Reviewed

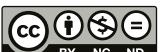
open 2 access

\section{AUTOR:}

(D) Bélgica Cecilia Arias Macías Universidad de Especialidades Espíritu Santo - Ecuador barias@uees.edu.ec

(1) Carmen Luisa Soto Montoya Universidad de Especialidades Espíritu Santo - Ecuador carmensoto@uees.edu.ec

\section{Conflicto de intereses}

Las autoras declaran que no existe conflicto de interés posible.

Financiamiento

No existió asistencia financiera de partes externas al presente artículo.

Agradecimiento

N/A.

Nota

El presente artículo no se desprende de un trabajo anterior.

ENTIDAD EDITORA 


\section{Introducción}

En la última década del siglo XX y a inicios del siglo XXI, emerge con fuerza el término "responsabilidad corporativa", también denominado "responsabilidad social empresarial". Este concepto engloba una serie de acciones emprendidas por las empresas para realizar sus actividades productivas de manera sostenible y sustentable. Toda empresa nace con la idea de permanecer en el mercado de manera indefinida, sin embargo, eso solo se logra con una adecuada administración y organización, con una planificación estratégica que genere una ventaja competitiva sobre sus similares. Esta estrategia debe alzar la mirada a la realidad del medio en el que la empresa se desenvuelve y ejecutar las acciones más apropiadas que permitan sobresalir sobre otras. Así tenemos que, es imprescindible que las empresas detecten y solucionen sus necesidades ordinarias para permanecer y crecer en el mercado, de esta manera atenderán los cambios que exige un mundo globalizado (Mora Castellanos et al., 2019).

Estrella \& Vives, (2011) consideran a la responsabilidad social corporativa como una forma de minimizar el impacto negativo en la sociedad y el medioambiente respetando al resto de los actores en la sociedad, contribuyendo a mejorar la calidad de vida de la población. Por consiguiente, Vives (2017) manifiesta que la responsabilidad social empresarial (RSE) es una decisión voluntaria que la empresa asume para contribuir al desarrollo social sustentable a través de un modelo de gestión planificado estratégicamente e involucrando a los stakeholders o grupos de interés. Por otro lado, Jaimes et al., (2021) sostienen que, su importancia surge cuando las empresas responsables socialmente son reconocidas a nivel mundial por los esfuerzos para minimizar las consecuencias negativas de su actividad empresarial en la comunidad y el medio ambiente.

En este sentido, la Comisión Económica para América Latina y el Caribe (CEPAL) de la Organización de las Naciones Unidas, indica que las empresas tienen varios caminos posibles para el desarrollo sostenible: 1) La responsabilidad social y ambiental; 2) Ecoeficiencia; 3) Producción más limpia, tecnológica y reciclaje; 4) Los sistemas de gestión (ISO 14000, análisis de ciclo de vida, contabilidad ambiental); 5) Pacto global y GRI (CEPAL, 2018). Las empresas del mundo, de Latinoamérica y en Ecuador han decidido seguir la ruta de la Responsabilidad Social Empresarial como una opción de competitividad y sostenibilidad (Vives \& Peinado-Vara, 2011).

En el Ecuador, se fortalece el criterio de que las actividades productivas busquen "producir, intercambiar y consumir bienes y servicios con responsabilidad social y ambiental" (Constitución Política de la República del Ecuador, 2008 Art.14). Ese es el caso de las instituciones financieras ecuatorianas que, si bien no realizan una actividad productiva directa en el país, son el motor que inyecta efectivo a la economía y posibilitan la instalación de negocios que mueven el mercado a través de la creación de empleos y mejora de la productividad. De tal manera que posibilitan la estabilidad financiera y la confianza de las personas que desean invertir y ahorrar.

Las instituciones financieras (bancos, cooperativas de ahorro y crédito, mutualistas, entre otras) sean públicas o privadas, son responsables socialmente cuando identifican situaciones ambientales, éticas y sociales en el diseño de los productos y servicios, en su gestión de crédito e inversión y en definitiva en todo su accionar (Sanchis \& Rodríguez, 2018). La RSC posee tres dimensiones principales: la económica que persigue la eficiencia y la productividad, (Ena \& Delgado, 2012); la social interna que involucra a los empleados en cuanto a inversión en recursos humanos, salud y seguridad, así como la social externa que se extiende a las comunidades locales e incluye 
socios comerciales y proveedores, consumidores, autoridades públicas y ONG defensoras de los intereses de las comunidades locales y el medio ambiente (Libro Verde, 2001); $y$ y, la medioambiental que comprende iniciativas que contribuyan a la reducción del impacto que tiene la actividad empresarial sobre el medio ambiente (Barrio, 2019).

Esos criterios han sido acogidos por las IF del Ecuador, quienes voluntariamente ejecutan estrategias de responsabilidad corporativa en sus acciones productivas. Por consiguiente, el objetivo de la presente investigación es determinar el grado de cumplimiento de la responsabilidad social corporativa en las instituciones financieras del Ecuador, en aras de contribuir al desarrollo sostenible local.

\section{Metodología}

El diseño del trabajo es no experimental de corte longitudinal. La investigación tiene un alcance descriptivo porque especifica propiedades, características y rasgos importantes de cualquier fenómeno que se analice. También es correlacional, debido a que la utilidad principal de este tipo de estudios es saber cómo se puede comportar un concepto o una variable al conocer el comportamiento de otras variables vinculadas ( Hernández et al., 2016).

Tabla 1. Metodología utilizada en la investigación

\begin{tabular}{|c|c|}
\hline Descriptivo & Correlacional \\
\hline $\begin{array}{l}\text { - } \quad \text { Mide y recoge información de manera independiente } \\
\text { - } \quad \text { y/o conjunta en las dimensiones de: } \\
\text { tomando en cuenta los balances de las tres instituciones } \\
\text { financieras anteriormente mencionadas. } \\
\text { - Social, se utilizó una encuesta realizada a } 15 \\
\text { colaboradores de diferentes áreas y cargos de las tres } \\
\text { instituciones. } \\
\text { - Ambiental, toma informes de sostenibilidad de la GRI } \\
4 \text { o llamadas también, memorias de responsabilidad } \\
\text { social, del año } 2019 \text { del Banco del Pacífico, Pichincha } \\
\text { y Produbanco. Posteriormente se compara los índices } \\
\text { correspondientes a la categoría Medio Ambiente. }\end{array}$ & $\begin{array}{l}\text { Porque las dimensiones son asociadas mediante Global } \\
\text { Reporting Initiative (GRI).y sus contenidos básicos generales. }\end{array}$ \\
\hline
\end{tabular}

Fuente: elaborada por las autoras.

Para el presente estudio, se seleccionó tres de las cuatro instituciones financieras consideradas como grandes por la Superintendencia de Bancos del Ecuador a través del método CAMEL, (ver tabla 2 y 3), por la similitud de aplicación de las GRI 4. Estas son: Banco del Pacífico que es el segundo banco con mayores utilidades del país, Banco Pichincha que es el banco más grande del Ecuador, además tiene presencia en Colombia, España, Panamá y Perú, y el Banco de la Producción con su nombre comercial Produbanco que se ubica como el tercer banco más grande. Cuenta con 101 agencias a nivel nacional. Se excluye de este estudio al Banco Guayaquil por no tener similitud de aplicación de las GRI 4, dado que esta institución realiza una evaluación interna y su formato no es comparable.

Tabla 2. Sistema de Bancos al 31 de diciembre del 2019

\begin{tabular}{|l|c|}
\hline \multicolumn{2}{|c|}{ Tamaño de instituciones Millones de dólares } \\
\hline BANCOS GRANDES & + de USD 1.000 millones \\
BANCOS MEDIANOS & entre USD 200 y USD 1.000 millones \\
BANCOS PEQUEÑOS & activos menores a USD 200 millones \\
\hline
\end{tabular}

Fuente: Superintendencia de Bancos 
Tabla 3. Clasificación de las Instituciones Financieras del Ecuador

\begin{tabular}{|c|c|c|}
\hline Orden & Institución & Tamaño de Instituciones \\
\hline 1 & B.P. Guayaquil & BANCOS GRANDES \\
\hline 2 & B.P. Pacífico & \\
\hline 3 & B.P. Pichincha & \\
\hline 4 & B.P. Produbanco & \\
\hline 5 & B.P. Austro & \\
\hline 6 & B.P. Bolivariano & \\
\hline 7 & B.P. Citibank & \\
\hline 8 & B.P. General Rumiñahui & BANCOS MEDIANOS \\
\hline 9 & B.P. Internacional & \\
\hline 10 & B.P. Loja & \\
\hline 11 & B.P. Machala & \\
\hline 12 & B.P. Solidario & \\
\hline 13 & B.P. Procredit & \\
\hline 14 & B.P. Amazonas & \\
\hline 15 & B.P. Comercial Manabí & \\
\hline 16 & B.P. Litoral & BANCOS PEQUEÑOS \\
\hline 17 & B.P. Coopnacional & \\
\hline 18 & B.P. Capital & \\
\hline 19 & B.P. Finca & \\
\hline 20 & B.P. Delbank & \\
\hline 21 & B.P. Demiro S.A. & \\
\hline 22 & B.P. Desarrollo & \\
\hline
\end{tabular}

Fuente: Superintendencia de Bancos y Seguros

Se procede a determinar la responsabilidad social corporativa a través de las dimensiones económica, social y medioambiental. En la categoría económica se efectuó la comparación de los balances financieros de los años 2018 y 2019 de las tres IF. En la categoría social, se realizó una encuesta (Ver Anexo 1) a los empleados de las agencias de las ciudades Guayaquil y Quevedo de estas IF con el fin de conocer su percepción sobre el ambiente laboral. A nivel nacional se estimó un tamaño de la población de 6000 colaboradores, según los datos de las memorias de responsabilidad de las tres instituciones. Del total de empleados se procede a calcular el muestreo. Se aplica una fórmula dando como resultado una muestra de 15 empleados a encuestas por cada institución financiera.

$$
\begin{gathered}
n=\left(z_{-} a^{\wedge} 2^{*} p^{*} q^{*} N\right) /\left(d^{\wedge} 2^{*}(N-1)+z_{-} a^{\wedge} 2^{*} p^{*} q\right) \\
d=0.25 z=1.96 p=q=0.50 \quad N=6.000 \\
n=15.33
\end{gathered}
$$

La encuesta, aplicada en septiembre del 2020, cuenta con indicadores cuantitativos y cualitativos, de eficiencia (financiera o ecológica), de intensidad (horas de formación por empleado, grado de satisfacción de la clientela, etc.). Esto permite ser lo más transparentes y normalizados posibles para realizar una comparativa entre instituciones a nivel nacional e internacional (Amaya \& Amaya, 2003). 
En relación a la categoría Medio Ambiente, se tomaron los datos de las memorias de responsabilidad social de las tres IF, publicadas a junio del 2020. Se procedió a realizar una lista de cotejo basado en la GRI 4. Los contenidos básicos específicos son los siguientes: desechos reciclados, reducción de consumo energía eléctrica, reducción de consumo de agua biodiversidad, emisión de sustancias que agotan el ozono, efluente y residuos, productos y servicios, cumplimiento regulatorio, transporte general, evaluación ambiental de los trabajadores, mecanismo de reclamación en materia ambiental (ver Anexo 2).

El método utilizado para recoger los datos, es de "Datos Secundarios" recolectados por otros investigadores. Según lo establece Hernández et al., (2016) implica la revisión de documentos, registros públicos y archivos físicos o electrónicos, tomado de las memorias de responsabilidad social publicadas en la web.

\section{Resultados}

Los resultados demuestran que las instituciones financieras sí han cumplido con la responsabilidad social, según las comparaciones basadas en los índices de las Global Reporting Initiative, en sus tres dimensiones: Económicas, Social y Ambiental. En lo económico se verifica que han generado valor para sus inversionistas, conforme lo señala (Fernández, 2005) esto se ve reflejado cuando la rentabilidad para los accionistas supera las expectativas mínimas sobre la inversión, que depende directamente de las estrategias operativas y financieras aplicadas (Pérez, 1998).

En relación a los datos obtenidos (Ver figura 1), se observa que el Banco Pichincha supera el patrimonio de las demás instituciones, siendo la entidad más grande con activos de USD 11.437 millones, destacándose de las demás instituciones por el índice de crecimiento en la cartera de créditos. Seguido está el Banco del Pacífico con USD 6.082 millones en activos, su mejor índice es de solvencia patrimonial. Posteriormente, Produbanco mantiene sus activos en USD 5.171 millones y sus indicadores financieros también son razonables.

Figura 1. Valor económico directo generado y distribuido $\mathrm{G}_{4}-\mathrm{EC}_{1}$

\begin{tabular}{|l|r|r|r|r|r|r|}
\hline & \multicolumn{2}{|c|}{ Banco del Pacifico } & \multicolumn{1}{c|}{ Produbanco (Grupo Promerica) } & \multicolumn{2}{c|}{ Banco Pichincha } \\
\hline & \multicolumn{1}{|c|}{$\mathbf{2 0 1 9}$} & \multicolumn{1}{c|}{$\mathbf{2 0 1 8}$} & \multicolumn{1}{c|}{$\mathbf{2 0 1 9}$} & \multicolumn{1}{c|}{$\mathbf{2 0 1 8}$} & \multicolumn{1}{c|}{$\mathbf{2 0 1 9}$} & \multicolumn{1}{c|}{$\mathbf{2 0 1 8}$} \\
\hline ACTIVO & 6.082 .137 & 5.533 .587 & 5.171 .926 & 4.768 .269 & 11.437 .419 & 10.664 .470 \\
\hline CARTERA DE CRÉDITOS & 4.089 .085 & 3.713 .577 & 3.296 .575 & 2.992 .168 & 6.962 .997 & 6.417 .709 \\
\hline PASIVOS & 5.314 .821 & 4.843 .898 & 4.709 .985 & 4.348 .348 & 10.175 .966 & 9.499 .995 \\
\hline OBLIGACIONES CON EL PÚBLICO & 4.453 .093 & 3.988 .924 & 4.023 .489 & 3.688 .404 & 8.990 .042 & 8.565 .950 \\
\hline PATRIMONIO & 767.316 & 689.689 & 461.941 & 419.921 & 1.261 .452 & 1.164 .474 \\
\hline
\end{tabular}

Fuente: Datos tomados de la Superintendencia de Bancos. (Cifras en millones de dólares).

En la categoría social, sobresale el Banco del Pacífico, al obtener mejores resultados en las encuestas realizadas. Sus colaboradores demuestran sentido de pertenencia, confianza, retribución económica, equidad, ambiente laboral disfrutable, innovación, flexibilidad laboral, comodidad del espacio físico y reciben capacitación continua. Esto se ratifica; al ser la institución que obtuvo el tercer lugar Great Place To Work 2019 (GPTW) que es el Ranking de las "Mejores empresas para Trabajar en Ecuador". 
De las memorias de responsabilidad social se extrae que, acorde a la G4-LA13 (Ver figura 2), existe igualdad de la retribución entre hombres y mujeres. Asimismo, los resultados más relevantes de la encuesta, muestran que los colaboradores perciben preocupación por su bienestar de parte de los directivos (Ver figura 3).

Figura 2. Igualdad de retribución entre mujeres y hombres G4-LA13

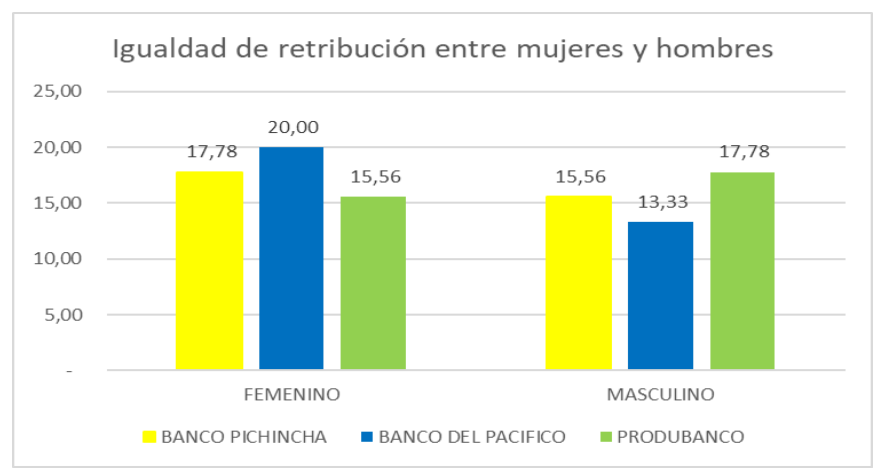

Fuente: Datos obtenidos de las memorias de responsabilidad social.

Figura 3. Seguridad y salud en el trabajo G4-LA5

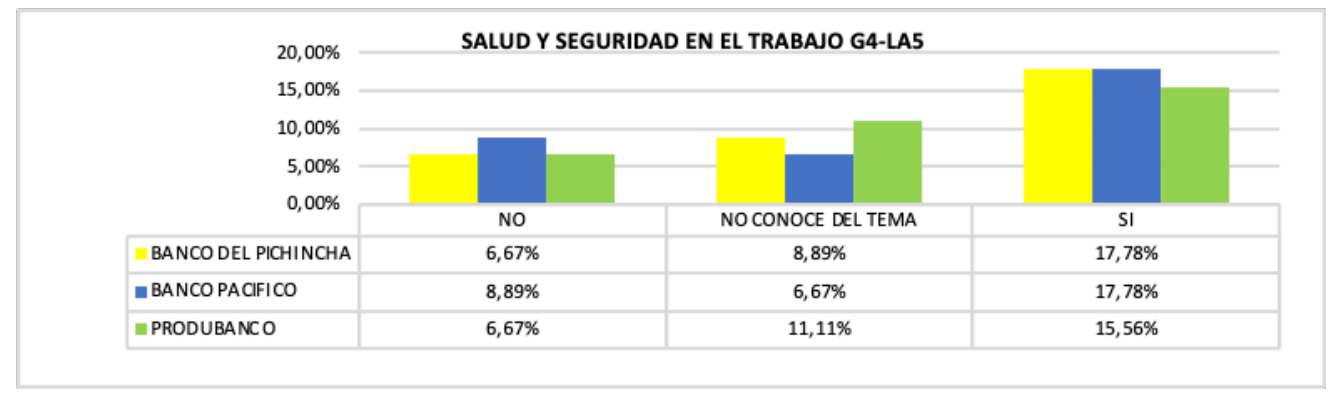

Fuente: Datos obtenidos de la encuesta para medir la dimensión social.

Por consiguiente, en cuanto a formación continua (Ver figura 4), se observan resultados favorables respecto a los indicadores G4-LAo9 que detalla horas de capacitación por sexo y categoría laboral; el G4-LA10 que evalúa los programas de formación continua y la gestión para que finalicen sus carreras y el G4-LA11 indica el porcentaje de empleados que han sido evaluados en su desempeño y evolución de su carrera. 
Figura 4. Capacitación y educación G4-LA9, LA10, LA11

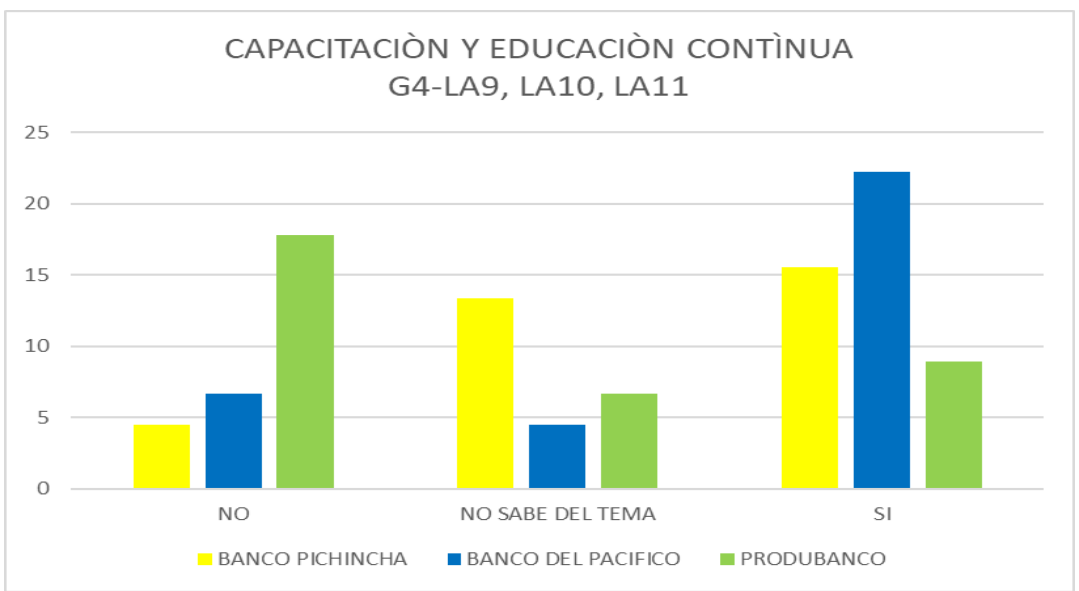

Fuente: Datos obtenidos de la encuesta para medir la dimensión social.

Finalmente, en la dimensión medio ambiente, los resultados de la lista de cotejo (Ver figura 5) muestran que Produbanco es la institución financiera que más cumple con los índices de medio ambiente conforme a los establecido en el Global Reporting Initiative. Se denota en proporción a los puntos específicos evaluados donde Produbanco, a pesar de tener el menor indicador económico, con respecto a esta norma, cumple en mayor porcentaje con un $42 \%$, muy por encima de Banco Pichincha que alcanza el 33\% y Banco del Pacífico con un 25\%.

Figura 5. Cumplimiento del índice Categoría Medio Ambiente

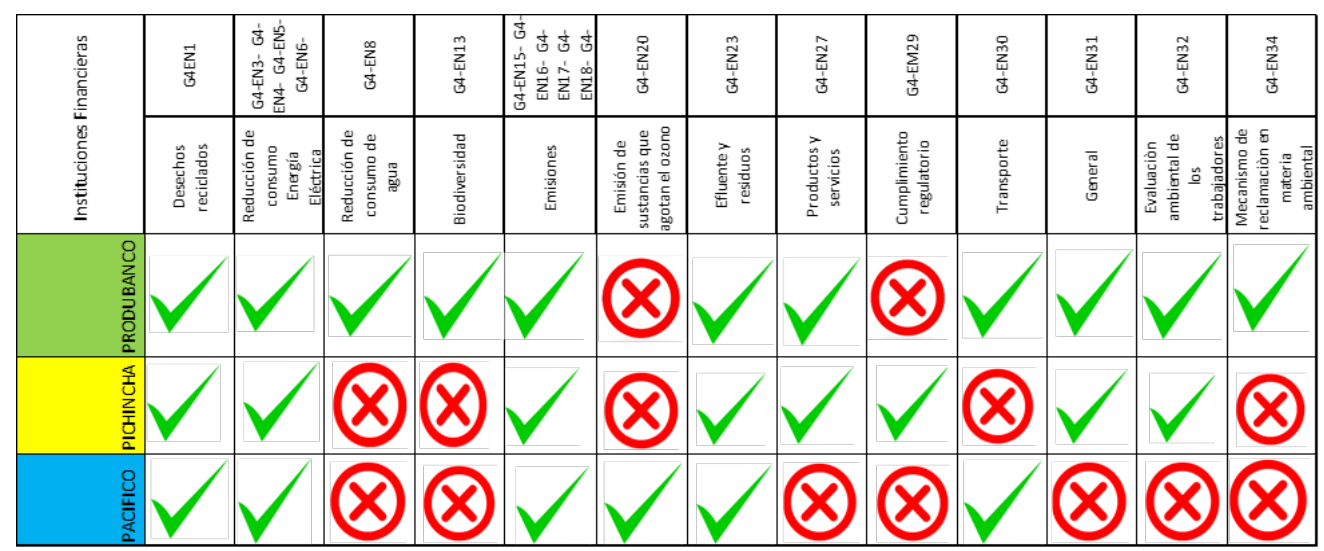

Fuente: Datos obtenidos de la lista de cotejo para medir la dimensión medio ambiente.

\section{Discusión}

Las tres instituciones estudiadas cumplen con la Responsabilidad Social Empresarial destacando en diferentes dimensiones. Así tenemos que, Banco Pichincha dirige sus acciones a maximizar su patrimonio. Su cartera de crédito muestra una diferencia significativa con otros bancos grandes y, por ende, su patrimonio es el más alto. Por otro lado, el Banco del Pacífico alcanza un impacto positivo en la sociedad. Es reconocido a nivel internacional como uno de los mejores lugares para trabajar, siendo esto corroborado por su talento humano. Busca el desarrollo profesional y personal de sus empleados, para, desde allí, posicionarse en la sociedad como un referente del trato justo 
e igualitario. Finalmente, Produbanco tiene como su fuerte la dimensión ambiental evidenciada en el cumplimiento de los índices de medio ambiente establecidos en el GRI, precautelando que sus actividades empresariales y el fomento productivo no impacten negativamente el medio ambiente.

Podría considerarse como limitantes de este trabajo, la población objeto de estudio. Sería interesante conocer las acciones emprendidas por diferentes empresas o instituciones del Ecuador, para cumplir con la RSC. Esto permitirá, además, identificar actividades que estén dando buenos resultados y que pueden ser replicadas por aquellas empresas que buscan ser responsables socialmente.

Estos hallazgos servirán de referente para que otras instituciones del Ecuador dirijan su acción productiva y empresarial a crecer económicamente, respetando la integridad humana tanto al interior como exterior de su institución y con la mirada fija en la naturaleza que es la fuente de donde extraemos todos los recursos para el bienestar y progreso humano y social.

\section{Conclusión}

La responsabilidad social corporativa es un indicador que conlleva a las empresas a actuar positivamente en varios aspectos, entre los que se destacan, el económico, el aspecto social y el medioambiental. Las instituciones financieras del país han trazado el camino para la RSC, visibles en los resultados publicados en sus memorias de responsabilidad social alineados a las GRI4 e ISO26000. Esta comparación ha llevado a determinar el punto de corte donde cada una de las instituciones analizadas muestran sus fortalezas. Las instituciones financieras son conscientes del resultado de su gestión en la economía del país y de su importancia en el fomento financiero, por ello, deben buscar el equilibrio entre la producción y el consumo.

\section{Referencias}

Amaya Navas, O. D., \& Amaya Arias, Á. M. (2003). Banca y medio ambiente Un ejercicio de responsabilidad social empresarial. Universidad Externado.

Barrio Fraile, E. (2019). Responsabilidad social corporativa. De la noción a la gestión. Questiones publicitarias 24, 103-103. https://raco.cat/index.php/questionespublicitarias/article/view/v2-n24-barrio

CEPAL. (2016). Horizontes 2030 La igualdad en el centro del desarrollo sostenible. Naciones Unidas.

Comisión de las Comunidades Europeas. (2001). Libro verde. COM

Constitución Política de la República del Ecuador. (2008, octubre 2008). Constitución Política de la República del Ecuador.

Ena Ventura, B., \& Delgado González, S. (2012). Recursos Humanos y Responsabilidad Social Corporativa. Paraninfo S.A.

Fernández, P. (2005). Creación de valor para los accionistas: definición y cuantificación. UCJC Business and Society Review (formerly Known As Universia Business Review), 2(6)10-25. https://journals.ucjc.edu/ubr/ article/view/526

Hernández Sampieri, R., Fernández Collado, C., \& Baptista Lucio, M. (2016). Metodología de la investigación. MCGRAW-HILL.

Jaimes Valdez, M. Á., Jacobo Hernández, C. A., \& Ochoa Jiménez, S. (2021). Los beneficios de la responsabilidad social empresarial: una revisión literaria. Tiempo \& economía, 8(2), 201-217. https://doi. org/10.21789/24222704.1720 
Mora Castellanos, C., Cano Olivos, P., Martínez Flores, J. L., \& Sánchez, D. (2019). De lo tradicional a un nuevo enfoque de microempresas: Modelo conceptual de alianzas estratégicas. Acta universitaria 29, 1-13. https://doi.org/10.15174/au.2019.2285

Palacio, S., Ramon, J., \& Rodríguez, S. (2018). Responsabilidad social empresarial en banca. Su aplicación al caso de la banca cooperativa. REVESCO: revista de estudios cooperativos 127, 204-227. https://doi. org/10.5209/REVE.59771

Vives, A. (2017). Una mirada crítica a la responsabilidad social de la empresa en Iberoamérica. Revista de Responsabilida Social de la Empresa, 161-164.

Vives, A., \& Peinado, E. (2011). La responsabilidad social de la empresa en América Latina. Fondo Multilateral de Inversiones (FOMIN).

\section{AUTORAS}

Bélgica Cecilia Arias Macías. Magíster en Contabilidad y Finanzas, de la Universidad de Especialidades Espíritu Santo. Contadora publica autorizada de la Universidad Técnica Estatal de Quevedo. Perito Contable acreditada por el Consejo de la Judicatura.

Carmen Luisa Soto Montoya. Magíster en Administración Pública, de la Universidad de Especialidades Espíritu Santo. Magíster en Educación Superior, de la Universidad de Guayaquil. 


\section{Anexos}

\section{Anexo 1: Encuesta \\ CATEGORÍA: DESEMPEÑO SOCIAL PRÁCTICAS LABORALES Y TRABAJO DIGNO}

Empleo G4-LA1 G4-LA2 G4-LA3; Relaciones entre los trabajadores y la dirección G4-LA4 Salud y seguridad en el trabajo G4-LA5 G4-LA6 G4-LA7 G4-LA8 Capacitación y educación G4-LA9 G4LA10 G4-LA11

1.- ¿Los directivos de la institución muestran preocupación por el bienestar de sus trabajadores en lo referente a salud, seguridad y familia?

$\mathrm{Si}$

No

No conoce del tema

2.- ¿La institución tiene delegada formalmente, una autoridad que se responsabilice por la difusión, capacitación y apoyo en la educación continua de sus colaboradores?

$\mathrm{Si}$ No

No conoce del tema

3.- ¿Considera usted que el clima laboral de la empresa es agradable?

$\mathrm{Si}$

No

No conoce del tema

4. ¿La institución le ofrece incentivos y crecimiento en el ámbito laboral?

$\mathrm{Si}$

No

No conoce del tema

5. ¿Se siente a gusto al trabajar para esta institución?

$\mathrm{Si}$

No 